\title{
Calidad, docencia y evaluación en la educación superior
}

\author{
Artículo a partir de conferencia en Congreso docente 19 enero 2014 \\ Universidad Tecnológica de El Salvador (Utec)
}

Víctor Manuel Gómez-Campo

Recibido: 02/01/2014 - Aceptado: 17/02/2014

\section{Resumen}

La calidad, docencia y evaluación en la educación superior son factores que se articulan en las actividades académicas universitarias, alrededor de un sistema de gestión de la calidad. La docencia es una función en que sus agentes y componentes deben obedecer a un sistema de calidad, el cual implica una evaluación permanente. La conferencia anual de docentes 2014, en la Utec, abordó aspectos relacionados con la premisa anterior, que en este artículo de reflexión se establecen como punto de partida el entorno y contexto de educación superior desde diferentes enfoques. La formación y el mundo del trabajo, que tienen relación con las 27 competencias plasmadas en Tunning América Latina, como vectores de calidad en la educación superior, el aprendizaje por proyectos y problemas, el reconocimiento y distinción al mérito y la creatividad. Formación por competencias, nuevos criterios, metodologías e indicadores de medición y evaluación de la calidad en la educación superior. Necesidad de establecer nuevos rankings sobre mejores prácticas, estatuto docente dual, aspectos que se deberían incluir en toda planificación educativa.

\section{Palabras clave}

Calidad, docencia, evaluación, mundo laboral, competencias.

\section{Abstract}

The quality, teaching and assessment in higher education are factors that are articulated in university academic activities around a system of quality management. Teaching is a function in which its agents and components must obey a quality system, which involves a continuing evaluation. The 2014 annual conference of teachers in the Utec, addressed issues related to the above premise, in this article reflection, establish as its starting point the setting and context of higher education from different approaches. The training and the world of work, dealing with the 27 competencies embodied in Tunning Latin America, as vectors of quality in higher education, learning through projects and problems \}, recognition and distinction to merit and creativity. Skills training, new methodologies and indicators measuring criteria and quality assessment in higher education. Need for new ranking on best practices, teaching dual status.

\section{Keywords}

Quality teaching, assessment, workplace, skills.

Víctor Manuel Gómez Campo. Doctor en Educación, con especialidad en Sociología de la Educación, Universidad de Massachusetts (Amherts). Pregrado en Sociología (B. A.). Tulane University (New Orleans). Profesor Asociado en la Facultad de Ciencias Humanas, Departamento de Sociología. Universidad Nacional de Colombia. Director, Maestría en Sociología. Departamento de Sociología. Director. Instituto de Investigación en Educación. Facultad de Ciencias Humanas. UN. Autor de varios estudios sobre Educación y Trabajo; Calificación y Formación; Educación Técnica y Tecnológica; Planeación Prospectiva en Educación; Informática en Educación; Diversificación Curricular en la Educación Media; Escuela Nueva y Educación Rural; Gobierno y Gobernabilidad en la Educación Superior; Formación Superior por Ciclos, Educación Media; Calidad en la Educación Superior. Ha sido consultor de diversos organismos internacionales como UNESCO, OIT, CINTERFOR, Instituto Internacional de Planeación de la Educación (IIPE-UNESCO), NUFFIC (Holanda). 


\section{Contexto de la educación superior (ES)}

Algunos de los componentes que se observan en el contexto de la educación superior son:

Masificación versus elitismo, movilidad social, inclusión (construcción de democracia), formación para la ciudadanía y la participación social y política; bildung versus razón instrumental. Lo extracurricular: la oferta cultural de formación general, de contextualización de la formación. Alta heterogeneidad sociocultural del cuerpo estudiantil; origen social y grado de 'capital cultural' (docentes). Politicas institucionales de selección, formación y promoción de docentes, estatuto docente, condiciones del trabajo docente, devaluación del pregrado, pertinencia social, cultural de la universidad.

\section{Contenido en el mundo del trabajo.}

Saberes, conocimientos, competencias (sociales, comunicativas, analíticas...); liderazgo. Aptitud al cambio, creatividad, innovación. Inestabilidad, obsolescencia. Alta competencia. Ciencia y tecnología, comercial, etc.; destrucción creativa (Schumpeter). La internacionalización. La sociedad del conocimiento. Sennett: la corrosión del carácter.

Consecuencias personales del trabajo en el nuevo capitalismo:

- Una importante dimensión de la calidad de la ES: las grandes diferencias existentes entre la calidad de las instituciones y la del aprendizaje logrado en los estudiantes (o de la calidad de la educación que efectivamente recibe el estudiante).

- La mayoría de los rankings internacionales y sistemas de acreditación privilegian indicadores de productividad e investigación, y otras dimensiones institucionales, siendo muy escasas las mediciones directas de la calidad de la educación que recibe el estudiante.

- La mayoría de indicadores de calidad de la ES son indirectos y derivados de indicadores de investigación o de nivel de formación del cuerpo docente.

- O son puntajes de exámenes (pruebas) artificiales de 'papel y lápiz', que por sus limitaciones metodológicas intrínsecas no pueden medir las competencias intelectuales y profesionales adquiridas.

- Necesidad de avanzar en criterios y procedimientos alternativos de medición y evaluación del aprendizaje logrado por los estudiantes o egresados del nivel superior. Logro de las competencias profesionales que se espera formar en la ES.

- Fuerte tendencia al mayor cambio de ocupaciones, oficios y trayectorias profesionales a lo largo de la vida laboral, estimulado por la diversidad de oportunidades de educación y recalificación continuas, y por la emergencia de nuevas ocupaciones, profesiones y especializaciones.

- Los itinerarios ocupacionales y educativos son cada vez más cambiantes y variados. Hay un alto grado de imprevisibilidad e indeterminación en la evolución del mercado de trabajo, de ocupaciones y profesiones.

Estos nuevos fenómenos en el mundo del trabajo cuestionan los criterios curriculares basados en perfiles o desempeños ocupacionales específicos. Plantean, además, la necesidad de nuevos criterios que conduzcan a revalorar el papel y la importancia curricular de la formación de competencias generales aún en los programas de estudio más especializados. Estos deben incluir, cada vez más, la formación de competencias para el aprendizaje y recalificación continuas, y para adaptabilidad a condiciones y exigencias cambiantes e imprevisibles.

En este contexto surge un primer interrogante acerca de ¿qué criterios, indicadores e instrumentos de medición se utilizan para la evaluación de la calidad de la educación superior? Que a su vez nos lleva a otras preguntas: ¿Se mide esta por la calidad de los egresados? ¿Por la calidad de la relación pedagógica? ¿Por el grado de homogeneización de condiciones de funcionamiento de las instituciones, según los criterios comunes de sistemas de acreditación? ¿Por la productividad de los docentes? ¿Por indicadores de investigación? ¿Por el lugar alcanzado en los rankings internacionales?

\section{Lo que lleva a reflexionar sobre el término calidad:}

- Para algunos, calidad significa el cumplimiento de determinados indicadores o parámetros comunes de funcionamiento de instituciones o programas. Homogeneización de condiciones académicas. Criterio común en algunos sistemas de acreditación.

- Dificultades para la evaluación positiva de innovaciones curriculares y pedagógicas. Ejemplo: Olin College of Engineering no sería 'acreditado'. 
- Para otros, el concepto de calidad hace referencia a logros y resultados en estudiantes, egresados y en la sociedad, independientemente de sus opciones curriculares y pedagógicas. Posibles experiencias innovadoras 0 heterodoxas.

Entonces, encontramos diversos conceptos de calidad de la ES y sus respectivos criterios y metodologías de evaluación.

a) Indicadores de productividad de docentes, indicadores de investigación.

- Los indicadores de productividad de docentes: de investigación, publicaciones, citas bibliográficas, publicación de revistas y libros, porcentaje de doctorados en el cuerpo docente.

- Los que demuestran y miden la productividad de los profesores en las universidades.

- Indicadores que forman rankings, jerarquizaciones, entre universidades y áreas del conocimiento. Necesidad de intenso recurso a la 'cienciometría', a sistemas internacionales de referencias bibliográficas y de autores.

- Los principales actores o interesados en este modelo de calidad de la ES son los profesores y directivos de la institución:

- Los estudiantes no desempeñan ningún papel, se los considera neutros o son invisibles.

- El interés particular de los profesores se deriva de los sistemas y criterios de estímulo, reconocimiento y remuneración a la productividad.

- su nivel de remuneración y su estatus académico dependen de su productividad.

- La mayoría aspira a publicaciones de categoría A y B, siendo subvaloradas publicaciones que pueden tener alta pertinencia social y valor político, pero que no son reconocidas ni jerarquizadas con valor académico.

- Un importante efecto: individualización del proyecto académico del profesor, pues su remuneración y promoción en el escalafón dependen de su puntaje individual de productividad.

- Otro efecto de gran importancia sobre la calidad de la ES es la invisibilización y marginalización de la función docente, la que no es reconocida ni valorada en los puntajes de productividad.

- La función docente es neutra en la remuneración y en el estatus académico.
En muchas universidades, "da exactamente lo mismo ser buen docente, mal docente o pésimo docente".

- Para las directivas, es importante que su institución esté bien ubicada en los rankings internacionales, fuente de estatus y distinción institucional. Se privilegia la productividad del profesor sobre su desempeño docente. Este no es reconocido ni valorado y no forma parte de los criterios de jerarquización internacional.

- Este modelo de calidad de la ES tiene como referente ideal los ejemplos internacionales de 'universidad de investigación' (research university).

- En estas universidades son mucho más importantes los postgrados que el pregrado, el que generalmente tiene problemas de baja calidad, pues la docencia en este nivel tiende a estar en manos de 'asistentes de docencia' (teaching assistants), estudiantes de postgrados 0 docentes temporales o de cátedra.

- Los profesores-investigadores, en doctorados, son relevados de la docencia en pregrado (Sexton, 2003).

b) Grado de cumplimiento de determinadas condiciones de funcionamiento y dotación (acreditación).

Grado de cumplimiento de determinadas condiciones homogéneas de funcionamiento y dotación: requisitos curriculares, de personal docente, infraestructura, dotación de bibliotecas y laboratorios o talleres, etc. En muchos países la acreditación de 'calidad' se basa en la demostración y verificación del logro de estas condiciones homogéneas.

- Este conjunto de condiciones de funcionamiento es definido y normalizado como el estándar ideal de calidad, el 'deber ser', al que todas las instituciones deben acercarse.

- Una importante limitación metodológica es que no se mide ni evalúa la calidad de la educación que reciben los estudiantes, ni la eficacia en el aprendizaje. Se utilizan indicadores 'indirectos' que se suponen estar positivamente asociados a la calidad de la educación.

- Pero estas asociaciones 'indirectas' no son válidas, como ha sido señalado en la literatura especializada internacional. (Barnett, 2011). 'Para una Transformación de la Universidad. Nuevas relaciones entre investigación, saber y docencia.'

- Ni el número o valor de las investigaciones, ni la productividad de los docentes, ni su perfil de formación, 
ni el número de premios Nobel en la institución están positivamente relacionados con la calidad de la educación que reciben los estudiantes de pregrado.

- Numerosas universidades de investigación (research universities) presentan grandes carencias en la calidad de la educación de pregrado, pues las prioridades de estas universidades residen en la función de investigación y en los doctorados.

- Un posible efecto perverso de este modelo (acreditación) es la existencia de instituciones que cumplan formalmente las condiciones e indicadores requeridos, pero sin cambios cualitativos en la pedagogía y la calidad de la educación que reciben los estudiantes.

c) Pruebas de conocimientos o competencias generales y profesionales según carreras o áreas de formación, al final de la carrera o últimos semestres.

En estas pruebas de 'papel y lápiz' es evidente la contradicción conceptual entre una supuesta evaluación de 'competencias' (disciplinarias y profesionales) y las limitaciones metodológicas de pruebas artificiales.

Las competencias se demuestran, se verifican, se despliegan, se ponen en escena, en la práctica: en proyectos, ensayos, investigaciones, experimentos, modelos, soluciones de problemas, no en pruebas artificiales y con graves limitaciones metodológicas.

- Efectos perversos: el efecto de preparación (efecto 'drilling' o de entrenamiento) por el que el resultado del entrenamiento es identificado y valorado como aprendizaje significativo y como indicador de calidad de la educación.

- Una mala facultad de ingeniería puede concentrar recursos en la preparación de sus estudiantes (drilling, training), sin que los puntajes obtenidos sean indicadores válidos de la calidad de la educación recibida. Igual sucede en otras áreas del conocimiento.

- Otro efecto perverso es el énfasis o sobrevaloración del tipo de conocimientos que conforman la prueba, subvalorando o minimizando otros conocimientos 0 competencias, de gran importancia en el desempeño ocupacional del egresado.

- Finalmente, los usos sociales de los puntajes de estas pruebas, utilizados como propaganda institucional y mercadeo de imagen, sin que este artificio se corresponda con la calidad de la educación en esa institución. d) Mediciones de logros demostrables en Ciencia y Tecnología.

Mediciones de logros demostrables en Ciencia y Tecnología: patentes, prototipos, modelos, soluciones de problemas:

- Grado de competitividad en concursos, licitaciones, proyectos.

- Papel protagónico del Estado -y de empresas e instituciones privadas - en la financiación y organización de este tipo de concursos competitivos de mérito. Ejemplos en robótica, diseño de nuevos materiales y de software, desarrollo de energías alternativas, biotecnologías, transporte, etc.

- Efectos altamente positivos en las universidades por la emulación entre facultades o grupos de investigación y por la activa participación de estudiantes.

\section{Ejemplo: NASA www.darpa.mil/grandchallenge}

Los principales actores, interesados y beneficiarios de este modelo son los profesores y estudiantes, participantes en grupos de investigación y experimentación.

- Este modelo genera una estrecha articulación entre investigación, experimentación, innovación y docencia.

- Los estudiantes participan activamente, desde el pregrado, en grupos y proyectos de I+D, en concursos, licitaciones, ferias de innovación, competencias locales y nacionales.

- Lo que promueve una alta motivación en el aprendizaje basado en proyectos (project-based learning), en lugar de la tradicional formación pasiva, basada en acumulación de conocimientos.

- En economías basadas en logros continuos en productividad y competitividad, necesidad de estimular y promover la creatividad e innovación en la ES.

- El mejor medio es ofrecer retos y recompensas y distinciones a estudiantes y programas, mediante su participación en concursos, competencias, licitaciones en diversas áreas del conocimiento.

- En la actual 'sociedad del conocimiento', de la ciencia y la tecnología, de la innovación y la creatividad; es necesario que los sistemas educativos, de ciencia y tecnología, y el sector productivo, diseñen estrategias de promoción, estímulo y distinción a la competencia e idoneidad (entendidas como logros, creatividad, 
desempeño, solución de problemas, proactividad), tanto en profesionales como en científicos, como en el conjunto de la fuerza laboral.

- A mayor desarrollo de las capacidades creativas de los individuos (en las artes, la literatura, la ciencia, la tecnología, la cirugía, el diseño, la gestión), mayor capital humano, mayor PIB per cápita, mayor riqueza, mayor desarrollo.

- En Corea, Japón y Estados Unidos, por ejemplo, son muy importantes los concursos y competencias, regionales y nacionales, entre colegios y entre universidades, en determinados campos de las ingenierías y tecnologías, como la robótica, telecomunicaciones, nuevos materiales, transporte, nuevas fuentes energéticas, biotecnologías, soluciones a determinados problemas locales o nacionales, etc.

- Los términos de referencia de estos concursos definen el nivel de competencia esperado. Este nivel aumenta continuamente en el tiempo de un concurso a otro debido al mayor conocimiento y experticia acumulados.

e) Medición directa de la calidad de la educación que reciben los estudiantes.

Otra opción metodológica, estrechamente relacionada con la anterior, es la medición directa de la calidad de la educación que reciben los estudiantes en pregrado.

Dimensión cualitativa que se evalúa en los resultados del proceso educativo:

- En el desarrollo de sus capacidades de conceptualización y abstracción (capacidad de análisis simbólico);

- En su capacidad de formulación de preguntas de investigación, y en su capacidad de experimentación;

- En sus competencias en la solución de problemas, y en su creatividad en su disciplina (científica, tecnológica, artística, humanística).

- Este tipo de evaluación requiere criterios y metodologías muy diferentes de las comúnmente utilizadas, las que solo indirectamente miden la calidad de la educación que efectivamente recibe el estudiante.

- En esta opción se busca una medición directa, por lo que el foco de análisis se centra en las tesis y trabajos de grado; en el desempeño ocupacional de graduados; en los proyectos de investigación elaborados; en los logros científicos, tecnológicos o artísticos demostrados (soluciones de problemas, ensayos, obras literarias o musicales, diseños, proyectos, etc.)

De las 27 competencias identificadas en el proyecto Tuning América Latina (2007), se seleccionan las 12 siguientes como las más importantes en la formación en cualquier disciplina o profesión, por su efecto positivo sobre el futuro desempeño ocupacional del egresado:

- capacidad de abstracción, análisis y síntesis;

- capacidad de aprender y actualizarse;

- capacidad para identificar, plantear y resolver problemas;

- capacidad crítica y autocrítica;

- capacidad de investigación;

- habilidades para buscar, procesar y analizar información;

- capacidad de comunicación oral y escrita;

- capacidad de aplicar los conocimientos en la práctica;

- capacidad de comunicación en un segundo idioma;

- habilidades en el uso de las tecnologías de la información;

- capacidad de trabajo en equipo; y

- capacidad para organizar y planificar el tiempo.

Entonces, ¿cómo se forman y desarrollan esas competencias o capacidades? Para ello se pueden identificar cuatro vectores de calidad en la ES:

A. Alto grado de flexibilidad curricular; validación de modalidades de aprendizaje alternativas a los cursos y asignaturas.

B. Aprendizaje por proyectos y problemas.

C. Sistemas de promoción y estímulo al mérito y la creatividad.

D. Nuevos criterios y modalidades de evaluación del aprendizaje.

Es importante establecer en qué estamos, ¿en aprendizaje o enseñanza?, por lo que, para dar respuesta a ello, se deberá estar claro en que:

- En un programa de formación comprometido con facilitar el desarrollo de estas competencias debe primar el principio pedagógico de que su principal propósito es promover y facilitar el 'aprendizaje' del estudiante; y su autonomía y creatividad; en lugar de la tradicional 'enseñanza' al estudiante, en la que se tiende a considerarlo como 'receptor' de una secuencia de cursos y asignaturas, al final de los cuales se supone que ha recibido el conocimiento disciplinario prescrito por el programa y puede ser 'graduado' y aceptado en la disciplina o profesión. 
- En el modelo de 'enseñanza' el actor principal y dominante es el docente y el programa de su asignatura, y el estudiante es el receptor pasivo de la secuencia de cursos del programa.

\section{A. Flexibilidad curricular}

Se encuentran acá tres dimensiones estrechamente relacionadas:

1. Que un porcentaje significativo de los cursos o créditos del programa sean electivos, con la finalidad de que cada estudiante pueda definir y optar por su propio perfil de formación, según sus intereses.

2. Que el estudiante efectivamente encuentre una amplia y diversa oferta de cursos y oportunidades de aprendizaje. Esto implica superar los estrechos límites del departamento, facultad e institución y tener acceso a múltiples ofertas de cursos en diferentes universidades en la ciudad. De esta manera el estudiante logra mayor enriquecimiento en su perfil de formación y mayor autonomía en sus decisiones formativas.

3. Validación de modalidades alternativas de aprendizaje. El aprendizaje se puede realizar a través de diversas modalidades muy diferentes a los cursos tradicionales, como proyectos de estudio independiente (individuales o grupales) con la asesoría y seguimiento de un docente, tutorías individuales, pasantías, entre otras.

- Es necesario superar la tradición del 'curso' o asignatura, como la única modalidad válida y reconocida de aprendizaje. Tradición que se deriva del concepto tradicional de que educar es 'enseñar', y que el profesor es quien enseña y evalúa lo aprendido.

- En universidades en las que se estimulan las modalidades alternativas de aprendizaje se ha evidenciado la enorme creatividad de los estudiantes, altamente motivados por su mayor autonomía en el aprendizaje.

\section{B. Aprendizaje por proyectos y problemas}

- El desarrollo de las competencias de indagación e investigación es una tarea que en instituciones educativas de calidad empieza desde los primeros grados, de tal manera que sus egresados ya han avanzado significativamente en el desarrollo de estas competencias.

- En instituciones en las que se implanta la pedagogía por proyectos, que es una aplicación del principio de la educación activa, son evidentes los logros de sus estudian- tes en la solución de problemas, la experimentación, el estudio sistemático de fenómenos naturales y sociales, su competitividad en ferias y concursos de ciencia y tecnología.

- En el nivel superior es necesario promover la identificación de temas e intereses de investigación en los estudiantes, desde el primer semestre, para que desde el principio de su experiencia formativa exploren e indaguen diversos temas y problemas de su área, superando así la pasividad frente a la enseñanza de cursos.

- Estos proyectos e iniciativas constituyen la principal motivación para el aprendizaje y la iniciación práctica o aplicada en conceptos, teorías y métodos de investigación.

- Algunos de estos temas pueden convertirse posteriormente en trabajos de grado maduros o en áreas o perfiles de especialización del estudiante.

- Estos proyectos e iniciativas pueden ser tanto individuales como grupales, lo que fomenta las competencias interpersonales tan indispensables en el desempeño ocupacional.

- Para validar y legitimar estos proyectos e iniciativas de los estudiantes, es necesario organizar instancias de presentación, análisis, discusión y retroalimentación académica, las que pueden asumir la forma de seminarios o 'semilleros' de investigación, con determinado número de créditos académicos.

\section{Reconocimiento y distinción al mérito y la creatividad}

- Los proyectos e iniciativas de los estudiantes, propuestos desde los primeros semestres, deben ser objeto de programas de fomento y reconocimiento (distinción) al mérito y a la creatividad, mediante concursos y eventos de presentación y premiación de los mejores proyectos en cada grado, lo que a su vez constituye una modalidad alternativa de evaluación.

- Esta es una práctica común en numerosas universidades comprometidas con la promoción de la creatividad de los estudiantes, y es fuente de motivación para el aprendizaje y la innovación. En algunos países existen fondos e instituciones del Estado, y de sectores y empresas privadas, orientados a la promoción y distinción de logros académicos en diversas áreas del conocimiento: artes, música, literatura, robótica, arquitectura, biotecnologías, ciencias naturales, etc. 


\section{Nuevos criterios y modalidades de evaluación}

- En lugar de pruebas convergentes, donde en el mejor de los casos se mide el grado de comprensión de determinado autor, concepto o teoría, se requieren modalidades de evaluación y medición de los logros o competencias demostradas por los estudiantes: proyectos, prototipos, soluciones a problemas, ensayos creativos, experimentos, avances en los proyectos iniciados desde los primeros semestres, etc.

- En estos logros de los estudiantes es posible evaluar si el proceso formativo ha sido eficaz en el desarrollo de las capacidades de conceptualización y abstracción (capacidad de análisis simbólico); de formulación de preguntas de investigación y en su capacidad de experimentación; de sus competencias en la solución de problemas, y de creatividad en su disciplina (científica, tecnológica, artística, humanística).

- Otras competencias, como la capacidad de comunicación en otro idioma y el manejo creativo de Ias TIC, requieren un decidido compromiso institucional.

- Es necesario familiarizar al estudiante con lecturas y conferencias en otros idiomas y con diversas iniciativas de búsqueda y sistematización de información, articuladas a los proyectos de los estudiantes, utilizando los recursos de acceso informático de la universidad a redes y bases bibliográficas internacionales.

A continuación, en la figura 1, se ilustran los factores vinculados con la formación por competencias.

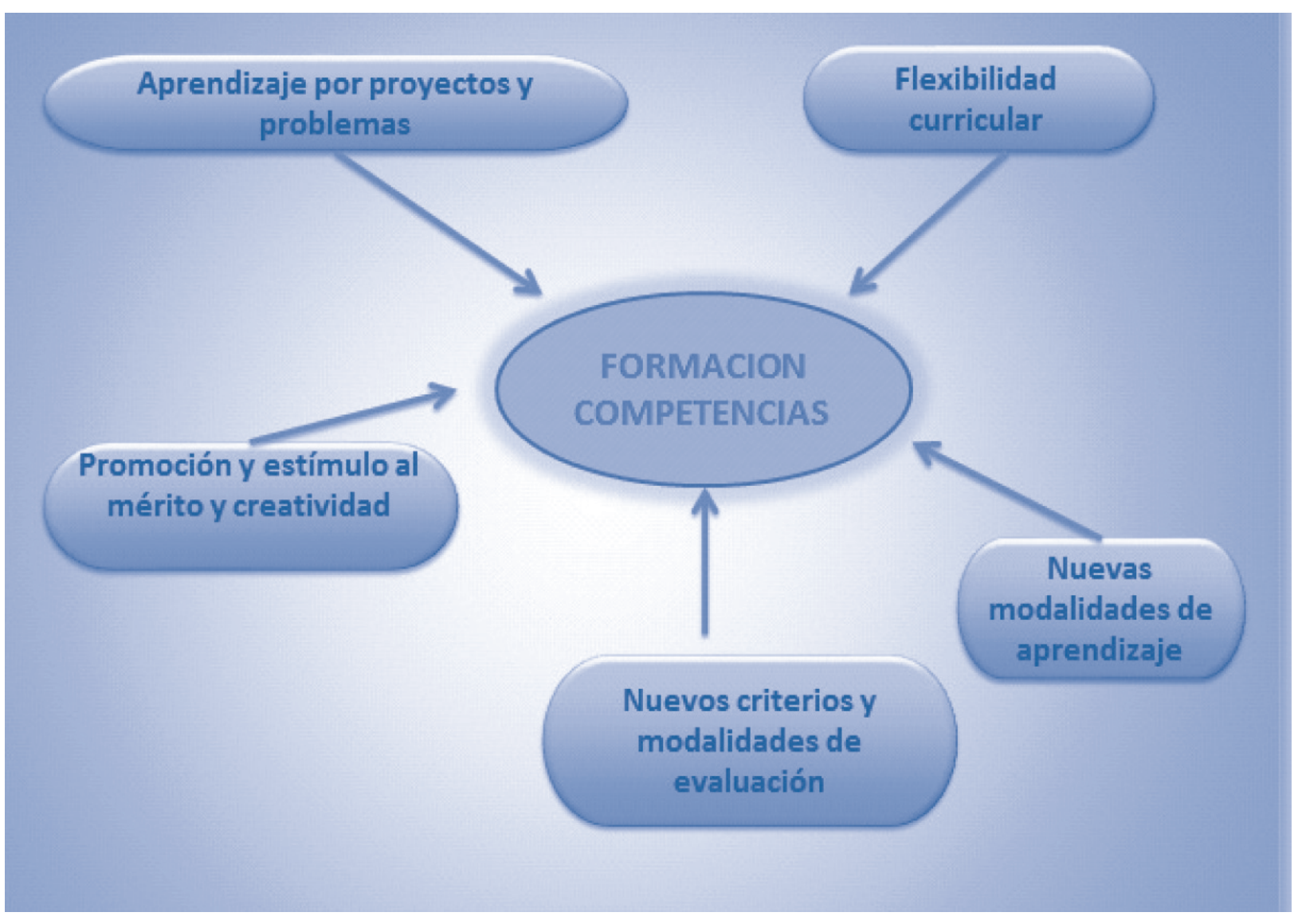

Figura 1

Ángulos en la formación por competencias.

Elaboración propia. 
No obstante, esta formación de competencias requiere:

1) Nuevos criterios, metodologías e indicadores de medición y evaluación de la calidad de la ES.

2) Análisis de la tensión entre el interés del Estado en medir, evaluar y establecer el ranking de la calidad de las instituciones; generalmente con metodologías estandarizadas; y la creatividad, diversidad e innovaciones en calidad, que no pueden ser medidas por pruebas estandarizadas.

3) Necesidad de diseño de nuevos rankings de calidad, basados en logros y resultados de la educación en estudiantes, egresados y la sociedad. Indicadores y parámetros de aprendizaje creativo y de pertinencia social de la universidad.

4) Desarrollo de sistemas de estímulos, promoción, distinción y estatus, a logros y competencias demostrados por estudiantes y profesores. Divulgación y emulación de 'mejores prácticas', como en: www.darpa.mil/grandchallenge.

5) Nuevos esquemas de estímulos, promoción, distinción y estatus a la 'función docente', la innovación pedagógica y didáctica de profesores, hoy subvalorada.

6) Estatuto docente dual, para el 'profesor-investigador' y para el profesor-docente (teaching professor), en donde se articulan en una sola función, y su planeación académica está vinculada con el quehacer docente e investigativo.

7) Esquemas de estímulo (promoción, remuneración) a las propuestas de investigación y a la productividad de docentes.

\section{Reflexión}

Estamos, entonces, ante grandes retos, en los que hay un compromiso de formar profesionales altamente competitivos que demanda el mundo laboral; un currículum pertinente y flexible que permita actualización de acuerdo con las exigencias del entorno; una planta de profesores que sea capaz de enseñar bajo un papel dual, con altas competencias en investigación, además de someterse a una actualización continua y generar productos de investigación que a la vez fortalezcan el proceso educativo, utilizando la investigación como una herramienta de aprendizaje. La institución educativa deberá evaluar la calidad de la enseñanza desde los resultados y productos de la investigación, colocación de graduados en el mundo laboral y al establecer un sistema de estímulos para profesores y estudiantes que permita no solo cumplir con los parámetros de las acreditaciones, sino también incidir en el entorno laboral y social, con profesionales competentes y sensibles ante la sociedad.

\section{Referencias}

Barnett, R. (2011). Being a University (Routledge).

Sexton, M. (2003). Portion of this dedication have been. Annual Survey of American Law. Volumen 60, Issue 1. March 27, 2003.

Tuning América Latina (2007). Reflexiones y perspectivas de la educación superior en América Latina. Informe final-Proyecto Tuning América Latina, Alfa III de la Unión Europea. Universidad de Deusto y Universidad de Groningena. Bilbao, España. 